\title{
THE EFFECT OF VARYING QUANTITIES OF INORGANIC IODIDE (CARRIER) ON THE URINARY EXCRETION AND THYROIDAL ACCUMULATION OF RADIOIODINE IN EXOPHTHALMIC GOITER ${ }^{1}$
}

\author{
By DONALD S. CHILDS, JR., F. RAYMOND KEATING, JR., J. EDWARD RALL,2 \\ MARVIN M. D. WILLIAMS, AND MARSCHELLE H. POWER \\ (From the Section on Therapeutic Radiology, Divisions of Medicine and Biochemistry, Mayo \\ Clinic, and Division of Physics and Biophysical Research, Mayo Foundation \\ and Mayo Clinic, Rochester, Minnesota)
}

(Submitted for publication December 19, 1949; accepted, February 27, 1950)

An ideal tracer experiment may be defined as one in which the quantity of labeled material added to the organism is too small to increase significantly the total quantity of the material which is already present within the body, and therefore is incapable of altering the metabolic equilibriums being studied. From this point of view an ideal tracer dose of $\mathrm{I}^{1313}$ in studies of thyroid physiology in man would be one which was carrier-free (that is, containing no added $\mathrm{I}^{127}$ ) and which contained the smallest quantity of radioactivity which the nature of the experiment and the sensitivity of the apparatus would permit.

Several investigators of thyroid function in the human have employed carrier-free doses of $\mathrm{I}^{131}$ usually in a dose of 100 microcuries. This quantity of radioiodine represents an extraordinarily minute amount of the element by weight $(8.1 \times$ $10^{-4}$ microgram) and, dissolved in $100 \mathrm{cc}$. of water, represents a $6.2 \times 10^{-11}$ molar solution. Because of concern over the possibility of significant losses by adsorption on glass surfaces from such extremely dilute solutions, we have preferred to employ for "tracer" doses 100 microcuries of $\mathrm{I}^{131}$ mixed with $0.1 \mathrm{mg}$. of stable iodide $\left(\mathrm{I}^{127}\right)=7.7 \times$ $10^{-7}$ molar solution. Such a dose falls short of the ideal conditions for a tracer study in that it does increase to an appreciable extent the total store of inorganic iodide in the body. It has the virtue of having approximately the magnitude of the

\footnotetext{
1 A portion of the material comprising this paper represents part of a thesis submitted by Dr. Childs to the Faculty of the Graduate School of the University of Minnesota in partial fulfillment of the requirements for the degree of Master of Science in Radiology.

2 Fellow in Medicine, Mayo Foundation.

${ }^{3} \mathrm{I}^{13}$ utilized in these studies was obtained from Oak Ridge National Laboratories, Oak Ridge, Tennessee.
}

minimal daily dietary increment of iodide (1) and for this reason it was hoped that it would not alter significantly the biologic equilibriums being investigated. The investigation here described was originally undertaken to evaluate this practical question: To what extent does carrier iodide affect the results of radioiodine studies in exophthalmic goiter? The results which have been observed throw light on several important aspects of thyroid function and raise a number of issues beyond the scope of the original question.

\section{METHODS}

The method employed was that of comparing successive tracer tests in the same subject, employing for each tracer different quantities of stable carrier iodide $\left(I^{127}\right)$. Such studies ideally should be carried out on normal or euthyroid subjects as well as on hyperthyroid ones. However, because of the possibility of some eventual deleterious effect on the thyroid gland resulting from the cumulative radiation of multiple tracer doses, we were unwilling to give multiple doses to euthyroid persons. Instead we have employed for this study patients with exophthalmic goiter who, for clinical reasons, had been selected for treatment with large doses of radioiodine. In these individuals, a series of successive tracer studies preceding radioiodine therapy was not regarded as hazardous. Patients with exophthalmic goiter proved particularly suitable subjects for this investigation, because their thyroidal function appears sensitive to small quantities of iodine and also because the marked increase in the iodine-accumulating function of the thyroid which they exhibited made it easier to show significant changes than would be the case with euthyroid persons.

Patients who had not been recently treated with antithyroid drugs or with iodine in any form were selected for study. Tracer doses were given at intervals of five days. In each instance the dose was given to the patient in the morning, breakfast being withheld. The urine was collected and its radioiodide content was measured as described previously (2). In some instances specimens of blood were withdrawn at various intervals and 
analyzed for radioactivity as described by McConahey and associates (3).

In vivo observations were made by two methods: 1 ) Frequent serial observations anterior to the thyroid were made with a Geiger counter for several days according to the technic described by Luellen and associates (4). Measurements were made with a shielded gamma ray counter centered $10 \mathrm{~cm}$. above the center of the thyroid gland. Each reading so obtained was corrected by a second reading, obtained immediately before or immediately afterward, with the counter tube in the same position but with a thick lead shield interposed between it and the thyroid. The second reading measured background from the rest of the body and was subtracted from the first reading to obtain a corrected value for counts recorded in the thyroid area. An additional correction for radioactivity in the tissues of the neck other than the thyroid was made by subtracting arbitrarily 2.5 times the corrected counts recorded $10 \mathrm{~cm}$. above the left thigh. 2) Measurement of the absolute quantity of radioiodine in the thyroid was made two or more times during each study by a method previously described by Keating and associates (5). For this purpose counts were obtained $25 \mathrm{~cm}$. and $45 \mathrm{~cm}$. above the center of the thyroid, each count was corrected for background with the lead shield interposed, as described earlier, and these observations were compared with a suitable standard of $I^{131}$. The inverse square relationship was employed to estimate the approximate effective point source of radiation. The $I^{121}$ content of the thyroid was calculated in microcuries or in per cent of administered dose. The average of such absolute determinations for each study was taken as a basis for a factor by which the entire curve of radioactivity obtained by the first method could be expressed in absolute terms.

In certain instances, $\mathrm{I}^{\mathrm{In}}$ was administered intravenously. The material to be given was diluted with physiologic saline solution, sealed in a glass ampule, and autoclaved. It was then withdrawn into a glass syringe encased in a specially constructed Lucite shield $1 \mathrm{~cm}$. thick. While such a shield affords little protection against the penetrating gamma rays of $I^{131}$ it was felt that it materially lessened the radiation hazard to the operator by increasing the distance of the gloved fingers from the source of radiation. By working efficiently and quickly, and performing no more than two such procedures a week, it was estimated that the total exposure to the fingers never exceeded and seldom approached the maximal permissible dose of 0.3 rep (roentgens-equivalent-physical) per week.

The urinary excretion curves obtained by plotting cumulatively the proportion of the dose excreted in the urine against time have been analyzed in the manner described by Keating and his associates (2). Such analysis yields the following values: 1 ) the renal fraction, defined as the asymptotic proportion of the dose excreted in the urine; 2) the disappearance rate, defined as the proportional rate at which inorganic radioiodide disappears from blood; 3 ) the renal excretion rate (a measure of renal function), defined as the proportional rate of excretion by the kidneys; and 4) the extrarenal disposal rate (designated as "collection rate" in the original publication), defined as the rate of disposal of radioiodine by all sites other than the kidneys. In so far as the thyroid is the principal extrarenal route of disposal of inorganic iodide, the extrarenal disposal rate has been employed as a measure of iodine-accumulating function of the thyroid gland and an index of thyroidal iodide clearance (5).

All tracer doses consisted of 100 microcuries of I's1 obtained from Oak Ridge National Laboratories. In seven patients a carrier-free ("0" mg.) dose of $\mathrm{I}^{131}$ was given; in the remaining six, the smallest dose given contained approximately $0.001 \mathrm{mg}$. of $\mathrm{I}^{22}$. All subjects were studied at two or three of the following levels of carrier iodide: $0.1,1,10,100$, and $500 \mathrm{mg}$. of $\mathrm{I}^{122}$. The order in which the various doses were given was altered in different cases so as to evaluate the possibility of a cumulative effect of radiation or of iodide from the preceding tracer tests. With one exception there was no evidence that the order in which the tracer tests were given had any effect. The exception was a study made five days after a test in which $500 \mathrm{mg}$. of $\mathrm{I}^{127}$ were used; the results were obviously influenced by residual iodide from the iodide of the first test and had to be discarded.

In nine cases a "control" study was done at the end of the series of tests, the study being repeated at the first carrier level investigated in each subject. In five of the nine subjects the values observed in this "control" study differed significantly from the initial one. The variations observed were of a random nature, however, and did not suggest a consistent effect which could be ascribed to the radiation or the iodide which had been received. In the case of extrarenal disposal rate, the most sensitive value derived from urinary observations, the mean difference for the nine cases was $+1.1 \pm 4.9$ per cent per hour, with a standard deviation of 15.6 per cent. Inasmuch as the time interval between initial and "control" observations was generally a month or longer, it would seem reasonable to ascribe such variation to changes in behavior of the disease per se rather than to the effect of radiation or of iodides.

Sixty determinations of serum $\mathrm{I}^{132}$ and $\mathrm{I}^{127}$ iodide were done in 11 studies of three patients and found to agree very well. Maximal levels of the order of 3 to 5 per cent of the dose per liter of serum were found; levels of $I^{127}$ iodide before administration of tracers were somewhat less than 1 microgram per $100 \mathrm{cc}$. On this basis the maximal serum iodide level after each of the doses employed may be estimated to be of the order of 1.0, 2.0, 2.5, 5.0, 50, 500, and 2,500 micrograms per $100 \mathrm{cc}$., respectively.

\section{RESULTS}

\section{Urinary observations}

The results observed in 51 series of observations on the urine of 13 patients with exophthalmic goiter are shown in Table I, and urinary excretion curves for a representative case (Case 13) are shown in Figure 1. All of the curves obtained 


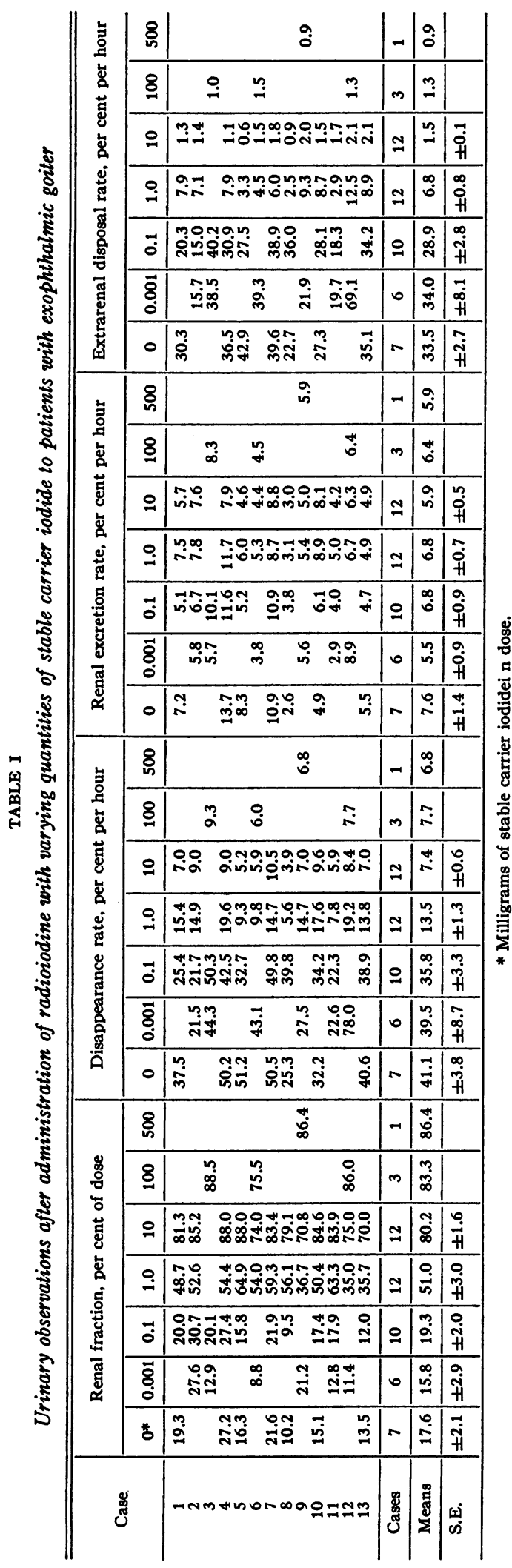

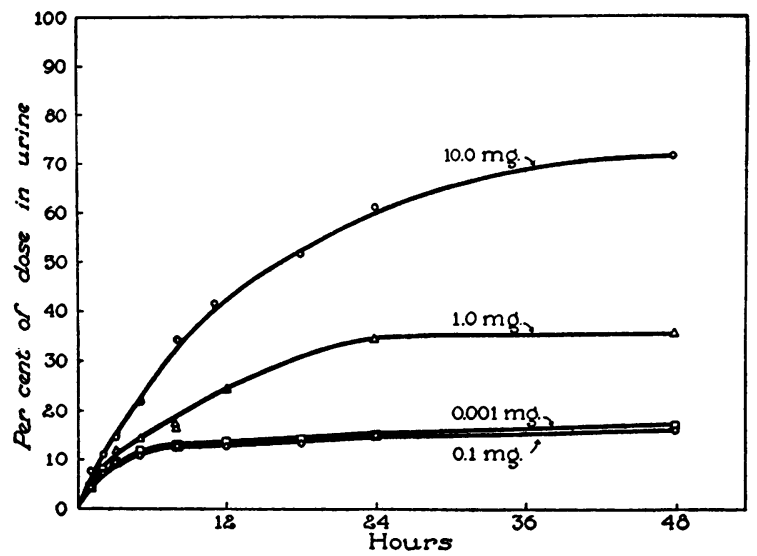

Fig. 1. Urinary Excretion Curves Obtained from the Same Patient with Exophthalmic Goiter at Different Times

The studies differ only with regard to the quantity of iodide administered, which is shown. Over the dosage range employed in this case, all of the patients studied behaved in the same way.

were essentially similar to the set shown. It can be seen that the form of the curves differs with increasing increments of iodide from a typically "hyperthyroid" pattern with the smallest doses of iodide to typically "hypothyroid" with the largest ones employed.

No significant difference appeared in the renal excretion rate with any of the various amounts of carrier used. Since this value is a measure of renal function with respect to iodide, it appears that the ability of the kidneys to excrete inorganic iodide is unaltered by changes in the quantity of iodide involved of the magnitude here employed.

In sharp contrast, however, are the observations of extrarenal disposal rate, which provides a measure of iodide disposal by tissues other than the kidneys. In cases of exophthalmic goiter, iodide disposal is predominantly via the thyroid. The mean values obtained without carrier are somewhat higher, but apparently not significantly higher, than those obtained with $0.1 \mathrm{mg}$. of carrier. In three of the cases values obtained without carrier appeared to be slightly, but significantly, higher than those obtained when $0.1 \mathrm{mg}$. of $\mathrm{I}^{\mathbf{1 2 7}}$ was used. In four cases the values did not appear to be significantly different. Similarly, the mean value obtained with $0.001 \mathrm{mg}$. of $\mathrm{I}^{127}$ was slightly but not significantly higher than that for $0.1 \mathrm{mg}$. On the other hand, comparison of extra- 
renal disposal rate with $0,0.001$ or $0.1 \mathrm{mg}$. and those obtained with $1.0 \mathrm{mg}$. or larger values shows very consistent and striking differences. Doses of $1.0 \mathrm{mg}$. of $\mathrm{I}^{127}$ produced a pattern of excretion and extrarenal disposal rates similar to those found in euthyroid individuals. With 10 mg. or more, there was consistently and uniformly an even greater change; for with that level the curves and rate values were indistinguishable from those of athyrotic persons.

The changes observed in the disappearance rates and the renal fractions reflect those of the extrarenal disposal rate, but less markedly. The interrelationships of those values have been discussed elsewhere (2).

The question whether the use of $0.1 \mathrm{mg}$. of $\mathrm{I}^{127}$ as carrier modifies the behavior of the $\mathrm{I}^{\mathbf{1 3 1}}$ tracer can be evaluated from a comparison of the urinary data in carrier-free and $0.1 \mathrm{mg}$. carrier studies in the seven patients receiving both doses. The mean differences for the latter compared to the former as standard are as follows: Renal fraction $+0.0 \pm$ 0.9 per cent, disappearance rate $-3.5 \pm 2.9$ per cent per hour, renal excretion rate $-0.8 \pm 0.7$ per cent per hour, and extrarenal disposal rate -2.6 \pm 2.4 per cent per hour. None of these mean differences appear significant. The possibility remains, however, that in an occasional unusually sensitive subject $0.1 \mathrm{mg}$. of iodide might modify the results.

\section{In Vivo observations}

Figure 2 illustrates representative in vivo thyroidal radioiodine curves obtained in a patient with exophthalmic goiter (Case 13) receiving four doses of labeled iodide at different times, the doses of $\mathrm{I}^{127}$ in this case being $0.001,0.1,1.0$, and $10.0 \mathrm{mg}$. of iodide respectively. It should be noted that the curves are of three types: 1 ) an increasing exponential curve (illustrated with 0.001 and $0.1 \mathrm{mg}$. of iodide) which reaches a maximum in about 12 hours, closely resembling in form the curves obtained from observations on urinary excretion of radioiodine; 2) an entirely different pattern, illustrated by the curve obtained after administration of $10.0 \mathrm{mg}$. of iodide, in which the thyroidal concentration of radioactivity rises to a peak value in approximately two and a half hours, and thereafter decreases more or less exponentially in a manner closely resembling the be- havior of radioiodine in the serum $(3) ; 3)$ a third type represented by the curve obtained after administration of $10.0 \mathrm{mg}$. of iodine, which appears to be a composite of the first two: an initial phase in which the thyroidal concentration rises rapidly to a peak in about three and a half hours and rapidly falls, and a second phase beginning after about six and a half hours, when a second exponential rise, resembling the response after administration of low carrier doses, takes place. All of the curves observed in all the cases could be divided among these three groups. In the description and discussion which follow, curves of the first type will be called "small-carrier curves" or iodine-accumulation curves; those of the second type "large-carrier curves" or inorganic iodidetrapping curves; those of the third type "intermediate-carrier curves" or mixed types. The basis for these particular terms will be discussed later.

It ishould be noted that in this particular case the 0.001 and $0.1 \mathrm{mg}$. curves are not identical, a larger proportion of the dose being accumulated when $0.1 \mathrm{mg}$. of carrier iodide $\left(\mathrm{I}^{127}\right)$ was given than when $0.001 \mathrm{mg}$. of carrier iodide was given. In other cases studied after these particular doses, the relationship was reversed; in still others, no significant difference appeared. Successive studies in the same patient with the same quantity of

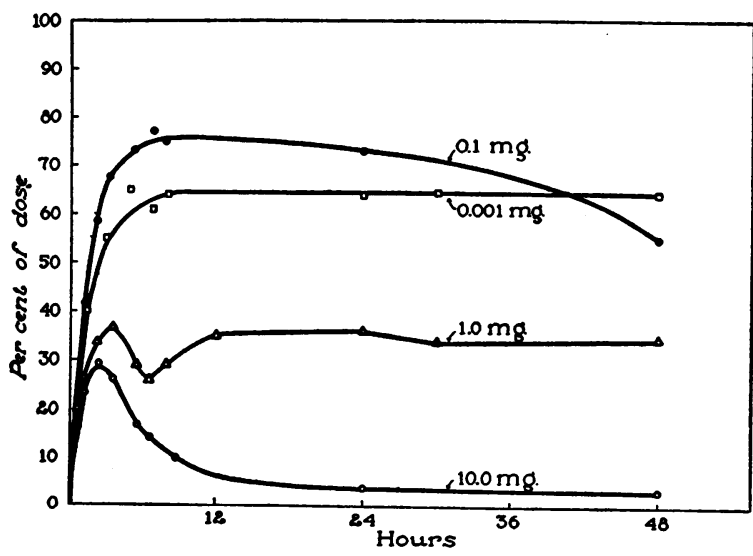

Fig. 2. In Vivo Observations of Radiolodine In THE Thyroid in a Case of Exophthalmic Gotter (Case 13)

Thyroidal curves following doses of $0.1,0.001,1.0$, and $10.0 \mathrm{mg}$. of $\mathrm{I}^{127}$ iodide are shown. These curves were obtained in the same case as the urinary curves shown in Figure 1. 
radioactivity and of carrier iodide have not infrequently disclosed similar variations in the results. Such differences have been assumed, therefore, to represent variation inherent in the fluctuating character of the diseased thyroid, the diet or other factors. We have not been able to show a consistent difference by in vivo methods in the thyroidal behavior of radioiodine between 0.001 $\mathrm{mg}$. and $0.1 \mathrm{mg}$. of carrier iodide.

Figure 3 illustrates a family of curves obtained in a second patient with exophthalmic goiter (Case 10 ), who received $0.1,0.75,1.0,2.0$, and $10.0 \mathrm{mg}$. of carrier iodide ( $\mathrm{I}^{127}$ ) respectively. As in the first case, the smallest dose shows a characteristic curve of the same type as the urinary excretion curve; the largest dose a characteristic curve of the same type as the serum curve, and the three intermediate curves appear to represent various degrees of admixture of the two extremes. Unlike the first case, however, the intermediate curves do not show a distinct secondary exponential phase.

Thirty studies of this sort have been carried out on seven of the 13 patients. The in vivo observations are summarized in Table II, in which for convenience the studies are grouped as "small carrier" when $0.1 \mathrm{mg}$. of $\mathrm{I}^{127}$ or less was used, "large carrier" when $10.0 \mathrm{mg}$. or more were used, and "intermediate" when 0.75 to $2.0 \mathrm{mg}$. were used. To conserve space no more than 10 observations during periods of 48 hours are given, although in

TABLE II

In vivo observations over the thyroid area after administration of varying quantities of iodide

\begin{tabular}{|c|c|c|c|c|c|c|c|c|c|c|c|}
\hline \multirow{2}{*}{ Case } & \multirow{2}{*}{$\begin{array}{c}\text { Dose } \\
\text { iodide, } \\
m g .\end{array}$} & \multicolumn{10}{|c|}{ Percentage of dose in thyroid gland after respective number of hours } \\
\hline & & $\frac{1}{2}$ & 1 & $1\}$ & 2 & 3 & 4 & 6 & 8 & 24 & 48 \\
\hline \multicolumn{12}{|c|}{ Small-carrier doses } \\
\hline $\begin{array}{l}3 \\
4 \\
5 \\
9\end{array}$ & $\begin{array}{l}0.001 \\
0.001 \\
0.1 \\
0.001 \\
0.1 \\
0.001 \\
0.001 \\
0.001 \\
0.1 \\
0.1 \\
0.001 \\
0.1\end{array}$ & $\begin{array}{l}16 \\
17 \\
15 \\
18 \\
30 \\
25 \\
27 \\
40 \\
18 \\
14 \\
16 \\
18\end{array}$ & $\begin{array}{l}24 \\
28 \\
25 \\
35 \\
44 \\
41 \\
42 \\
52 \\
40 \\
40 \\
27 \\
38\end{array}$ & $\begin{array}{l}28 \\
35 \\
45 \\
50 \\
47 \\
55 \\
62 \\
54 \\
49 \\
40 \\
47\end{array}$ & $\begin{array}{l}36 \\
42 \\
42 \\
50 \\
53 \\
57 \\
62 \\
68 \\
68 \\
54 \\
56\end{array}$ & $\begin{array}{l}42 \\
49 \\
49 \\
61 \\
63 \\
66 \\
70 \\
76 \\
71 \\
60 \\
55 \\
66\end{array}$ & $\begin{array}{l}44 \\
55 \\
54 \\
67 \\
71 \\
74 \\
83 \\
81 \\
63 \\
60 \\
71\end{array}$ & $\begin{array}{l}47 \\
59 \\
58 \\
76 \\
71 \\
80 \\
77 \\
84 \\
77 \\
68 \\
62 \\
75\end{array}$ & $\begin{array}{l}48 \\
62 \\
62 \\
75 \\
73 \\
82 \\
76 \\
82 \\
74 \\
72 \\
64 \\
75\end{array}$ & $\begin{array}{l}49 \\
63 \\
64 \\
73 \\
69 \\
80 \\
76 \\
85 \\
77 \\
72 \\
64 \\
73\end{array}$ & $\begin{array}{l}48 \\
50 \\
66 \\
68 \\
75 \\
79 \\
\\
64 \\
55\end{array}$ \\
\hline
\end{tabular}

Large-carrier doses

\begin{tabular}{|c|c|c|c|c|c|c|c|c|c|c|c|}
\hline $\begin{array}{r}3 \\
4 \\
5 \\
8 \\
9 \\
10 \\
13\end{array}$ & $\begin{array}{r}10.0 \\
10.0 \\
10.0 \\
100.0 \\
10.0 \\
500.0 \\
10.0 \\
10.0 \\
10.0\end{array}$ & $\begin{array}{c}12 \\
19 \\
9.0 \\
11.0 \\
16 \\
8.0 \\
15 \\
\frac{14}{14}\end{array}$ & $\begin{array}{l}16 \\
27 \\
10.0 \\
12.0 \\
25 \\
9.0 \\
18 \\
21 \\
19\end{array}$ & $\begin{array}{c}15 \\
34 \\
10.0 \\
11.0 \\
27 \\
8.0 \\
18 \\
25 \\
23\end{array}$ & $\begin{array}{c}14 \\
36 \\
10.0 \\
10.5 \\
25 \\
7.0 \\
17 \\
29 \\
28\end{array}$ & $\begin{array}{l}11 \\
34 \\
10.2 \\
10.0 \\
24 \\
7.0 \\
15 \\
33 \\
25\end{array}$ & $\begin{array}{c}11 \\
9.0 \\
10.0 \\
25 \\
5.8 \\
14 \\
30 \\
22\end{array}$ & $\begin{array}{c}8 \\
26 \\
14.0 \\
9.2 \\
22 \\
5.0 \\
11 \\
26 \\
16\end{array}$ & $\begin{array}{c}9 \\
27 \\
14.0 \\
8.0 \\
20 \\
-9 \\
25 \\
13\end{array}$ & $\begin{array}{c}0 \\
10 \\
9.0 \\
5.5 \\
11 \\
4.0 \\
2 \\
12 \\
4\end{array}$ & $\begin{array}{c}\overline{4} \\
10.0 \\
3.5 \\
8 \\
\frac{0.5}{6} \\
-\end{array}$ \\
\hline
\end{tabular}

Intermediate doses

\begin{tabular}{|c|c|c|c|c|c|c|c|c|c|c|c|}
\hline $\begin{array}{r}3 \\
4 \\
5 \\
8 \\
9 \\
10 \\
\\
13\end{array}$ & $\begin{array}{l}1.0 \\
1.0 \\
1.0 \\
1.0 \\
1.0 \\
0.75 \\
1.0 \\
2.0 \\
1.0\end{array}$ & $\begin{array}{l}-23 \\
18 \\
26 \\
22 \\
24 \\
20 \\
\frac{20}{20}\end{array}$ & $\begin{array}{l}14 \\
22 \\
29 \\
27 \\
35 \\
\overline{25} \\
27\end{array}$ & $\begin{array}{l}15 \\
33 \\
23 \\
33 \\
43 \\
36 \\
32 \\
30\end{array}$ & $\begin{array}{l}16 \\
43 \\
23 \\
33 \\
29 \\
42 \\
38 \\
35 \\
32\end{array}$ & $\begin{array}{l}17 \\
22 \\
34 \\
25 \\
45 \\
40 \\
39 \\
37\end{array}$ & $\begin{array}{l}18 \\
43 \\
21 \\
34 \\
25 \\
43 \\
40 \\
39 \\
34\end{array}$ & $\begin{array}{l}22 \\
36 \\
22 \\
33 \\
30 \\
41 \\
37 \\
34 \\
26\end{array}$ & $\begin{array}{l}24 \\
40 \\
22 \\
32 \\
37 \\
40 \\
35 \\
29 \\
29\end{array}$ & $\begin{array}{l}48 \\
26 \\
36 \\
47 \\
42 \\
38 \\
34 \\
20 \\
37\end{array}$ & $\begin{array}{l}45 \\
21 \\
40 \\
45 \\
31 \\
35 \\
33 \\
20 \\
36\end{array}$ \\
\hline
\end{tabular}




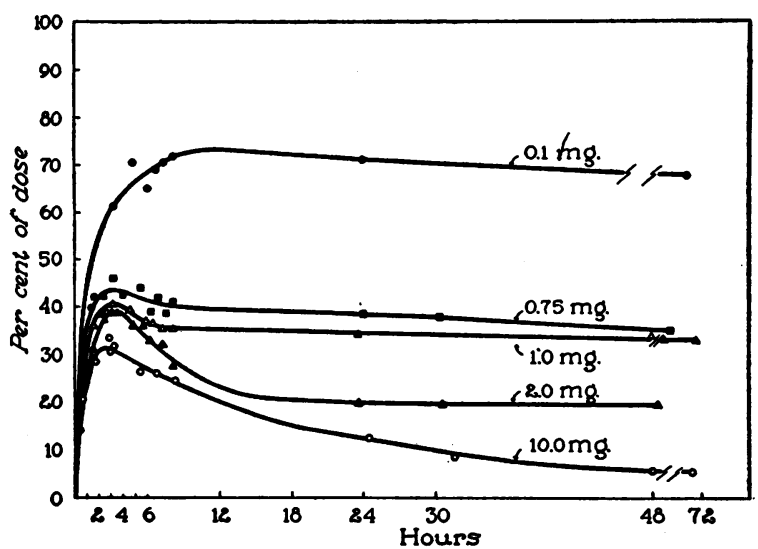

Fig. 3. In Vivo Observations of Radiolodine in a Second Case (Case 10)

In this instance levels of $\mathrm{I}^{127}$ iodide of $0.1,0.75,1.0,2.0$, and $10.0 \mathrm{mg}$. were given. The intermediate curves show a progressive change with dose and in this case do not have exactly the same form as the intermediate $(1.0 \mathrm{mg}$.) curve in Figure 2.

nearly all cases 20 or more observations were obtained in that period. The data for small-carrier doses all follow a constant pattern. Similarly the data for large-carrier doses also follow a constant pattern with the exception of the observations following a $10.0 \mathrm{mg}$. dose in Case 5 , which resemble an intermediate response. On the other hand the results obtained with $1.0 \mathrm{mg}$. iodide vary conspicuously from case to case. In one instance (Case 3) an initial rise is followed by a plateau and later a secondary rise. In others (Cases 4 and 10) the form of the intermediate curve is similar to that depicted in Figure 3. In still others (Cases 5, 8, 9, and 13) the form of the intermediate curve is similar to that in Figure 2; that is, an initial rise and fall, resembling the large-carrier curve, followed by a secondary rise, probably exponential, which is sustained, resembling the small-carrier curve.

\section{COMMENT}

\section{Urinary observations}

The lack of any significant change in renal excretion rate of $\mathrm{I}^{131}$ with widely varying quantities of the stable iodide as carrier deserves comment. As noted elsewhere (6), this value is related to renal clearance of iodide, being, in fact, clearance divided by volume of distribution. In five of the 13 cases, blood levels were obtained, permitting evaluation of renal iodide clearances by the conventional method. These are shown in Table III and likewise fail to show any significant change with altered quantities of iodide.

The values for iodide clearance are consistent with the view that iodide is filtered through the glomerulus and reabsorbed through the renal tubules. In situations (for example, urea) in which clearance is not significantly altered by wide variations in plasma concentration, the assumption may be made that the process of reabsorption by the tubules is a passive one not requiring osmotic work. However, with the quantities of iodide here employed, the blood concentration of iodide, by comparison with other anions, as for example chloride, is so very small as to make it probable that even if reabsorption did require osmotic work only a trivial expenditure of energy would be required at any of the levels observed. This matter was further tested in two patients with exophthalmic goiter by administration of doses of $500 \mathrm{mg}$. and in one subject by administration of a dose of $2.0 \mathrm{gm}$. of sodium iodide. These doses have been estimated to represent maximal blood concentrations of the order of $3 \mathrm{mg}$. per 100 cc. and $10 \mathrm{mg}$. per $100 \mathrm{cc}$, respectively. However, the renal excretion rate or clearance was not appreciably altered as compared with the values after administration of tracers containing only $0.1 \mathrm{mg}$. of iodide. Within these limits $(0.001$ to 2,000 mg.) the dose of iodide appears to have no effect upon the proportional renal excretion rate of iodide.

The contrast between the foregoing and the behavior of the thyroid as reflected by extrarenal disposal rate (Figure 4) is, as one would expect, most striking. In most of the instances studied in the range 0 to $0.1 \mathrm{mg}$., the dose of $\mathrm{I}^{127}$ made no difference in the results; in a few cases a slight

TABLE III

Renal clearance of radioiodine

\begin{tabular}{c|c|c|c|c|c|c}
\hline \hline $\begin{array}{c}\text { Carrier } \\
\text { I127, } \\
m g .\end{array}$ & \multicolumn{3}{|c|}{ Clearance of I's1, cc. serum per minute } & \multirow{2}{*}{$\begin{array}{c}\text { Mean and } \\
\text { S.E. }\end{array}$} \\
\cline { 2 - 5 } & Case 3 & Case 4 & Case 9 & Case 10 & Case 13 & \\
\hline 0.001 & 49.3 & 45.7 & 40.4 & & 41.7 & $44.3 \pm 2.0$ \\
0.1 & 35.0 & 38.4 & 53.0 & 32.7 & 25.5 & $36.9 \pm 4.5$ \\
1.0 & 37.2 & 51.6 & 36.5 & 29.2 & 16.9 & $34.3 \pm 5.6$ \\
10.0 & 45.6 & 33.8 & 51.6 & 25.0 & 29.6 & $37.1 \pm 4.9$ \\
\hline
\end{tabular}


change may have occurred with $0.1 \mathrm{mg}$. of $\mathrm{I}^{127}$. One milligram reduced the extrarenal disposal rate significantly in all cases, but in none did this dose produce values consistent with apparent total suppression of thyroidal iodide accumulation. With $10 \mathrm{mg}$. or more the values obtained suggest more or less complete suppression of thyroidal accumulating function in all cases. As will be discussed elsewhere, this "suppression" may be more apparent than real, for with the method used it could be possible for more $\mathrm{I}^{127}$ to enter the thyroid after administration of $10 \mathrm{mg}$. than after administration of $0.1 \mathrm{mg}$. and still be too small a proportion of the total to be detected.

Changes in the value for extrarenal disposal rate can, of course, result from alterations elsewhere than in the thyroid, since this value measures all tissues other than the kidneys. However, in patients with exophthalmic goiter the extrarenal disposal rate is predominantly due to increased thyroidal accumulation, and that part of it which can be accounted for by other tissues is of the order of 1 per cent per hour. The changes here observed can, therefore, represent only suppression or reduction of thyroidal function, a conclusion which is borne out by the direct in vivo studies. It is not possible with this indirect method, however, to determine whether or not a large quantity of iodide completely suppresses the

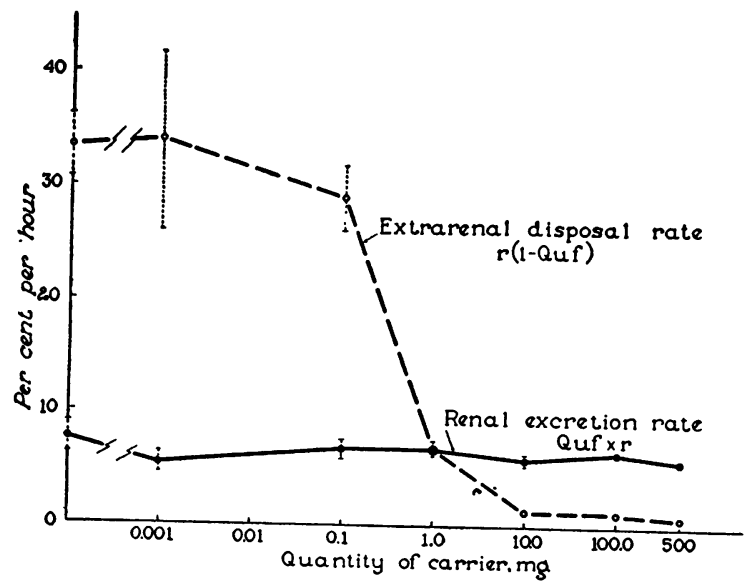

Fig. 4. Comparison of the Effect in Patients with EXophthalmic GoITER OF VARYing QUANTITIES OF Labeled InORganic Iodide Administered BY Mouth Upon the Renal Excretion of Iodide (as Evaluated by the Renal Excretion Rate) and the Thyroidal AcCUMulation of Iodide (as Evaluated Indirectly BY the Extrarenal Disposal. Rate) accumulation of iodide in the thyroid. With the larger doses a significant quantity of iodine could enter the gland and be quite undetectable by this means.

\section{In Vivo curves}

The contrasting types of behavior of radioiodine in the thyroid gland of patients with exophthalmic goiter, represented by the "small-carrier curves" and "large-carrier curves," have been previously noted. Hamilton and Soley (7) in 1940 published the first report dealing with in vivo observations of radioiodine in the human thyroid. They employed $14 \mathrm{mg}$. of inorganic $\mathrm{I}^{127}$ iodide as carrier. In normal control subjects the curves for thyroidal content of radioiodine showed a gradual exponential increase to a maximal level in 48 hours. Curves for patients with nontoxic goiters showed similar patterns, but the maximal concentration reached was greater than for the normals. The curves for five patients with thyrotoxicosis, however, were of markedly different shape, rising to a peak exceeding the maximal normal concentration within four hours, and thereafter decreasing rapidly to about one-fifth of the maximal values. In 1942 Hamilton (8) reported further observations on thyrotoxic subjects using 0.1 microgram of iodide as carrier. With this dose the thyroidal uptake curves observed in thyrotoxic patients were similar in shape to those previously observed in euthyroid subjects with $14 \mathrm{mg}$. of carrier iodide, but thyroidal accumulation of iodide was found to be much greater in thyrotoxic than in euthyroid subjects.

Curves similar to our large-carrier curves were obtained by Stanley and Astwood (9) in both normal and euthyroid human subjects by inhibiting the biosynthetic function of the thyroid with mercaptoimidazol prior to the administration of radioiodine. Under these conditions, in vivo studies disclosed an accumulation of radioiodine in the thyroid which reached a maximum one and a half to two and a half hours after administration of the dose, after which a rapid fall took place. Administration of potassium thiocyanate or of potassium iodide in a dose of $1 \mathrm{gm}$. some hours after administration of $\mathrm{I}^{131}$ caused a rapid discharge of the radioiodide concentrated in the thyroid.

More detailed observations of these phenomena 
have been made in experimental animals. Vanderlaan and Vanderlaan (10) demonstrated in 1947 that the thyroid of the rat, when normal synthesis was blocked by the prior administration of propylthiouracil, retained the capacity to concentrate iodine. They showed by ultrafiltration and potentiometric methods that the iodine so concentrated in the thyroid under these conditions remained in the form of inorganic iodide. In normal rats the ratio of thyroidal inorganic iodide to blood inorganic iodide was found to be 25 ; in rats previously made goitrous with propylthiouracil, the ratio was 250 . Injection of potassium thiocyanate resulted in immediate discharge of the inorganic iodide concentrated in the blocked thyroid. Taurog, Chaikoff, and Feller (11), almost simul-

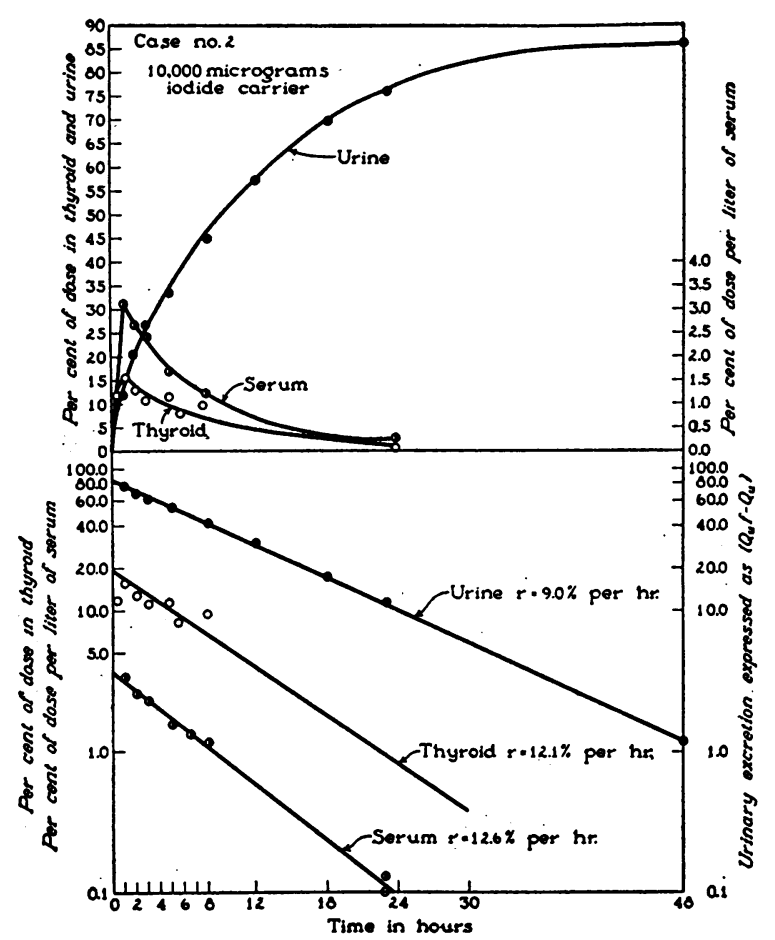

Fig. 5. Comparison of Serum Radioiodide Concentration, Urinary Excretion Curve and Thyroidal Curve in a Patient with Exophthalmic Goiter Given 10.0 MG. OF CARRIER IODIDE I ${ }^{127}$

The similarity between thyroid and serum curves is obvious; plotted on semilogarithmic paper the slopes are approximately parallel. Note that the peak value of the thyroid curve occurs some time later than the peak value of the serum curve; this was invariably the case and will be discussed elsewhere. Note also that the ebb and flow of radioiodine in the thyroid is not reflected by any apparent change in the urinary curve.
TABLE IV

Disappearance rate calculated from urine, thyroid and blood curves after administration of radioiodine with $10 \mathrm{mg}$. I ${ }^{127}$ iodide as carrier

\begin{tabular}{c|c|c|c|c}
\hline \multirow{2}{*}{ Case } & \multicolumn{4}{|c}{ Disappearance rate as per cent per hour } \\
Calculated from
\end{tabular}

taneously with the foregoing, published a paper detailing very similar observations. They found that the blocked-thyroid-to-serum ratio in goitrous rats was 220 to 270 and observed also that the disappearance of labeled inorganic iodide from the blocked thyroid paralleled the exponential decline of labeled inorganic iodide in the plasma.

These studies indicate that the thyroid has a specific function of concentrating inorganic iodide within the gland, that this function is ordinarily obscured by the very rapid biosynthesis by which inorganic iodide is combined to form thyroid hormone unless thyroidal biosynthesis is first suppressed, that the iodide-trapping function is promptly poisoned or abolished by administration of thiocyanate, and that the inorganic iodide concentrated or trapped by the thyroid remains in some sort of equilibrium with that in the blood.

Our observations with varying quantities of labeled iodide in patients with exophthalmic goiter suggest that inorganic iodide itself suppressed or obscured the relative biosynthetic function of the thyroid and that the curve of radioiodine concentration in the thyroid after administration of large doses of iodide reflects the iodide-trapping function of the thyroid in the same manner as did studies on thyroids blocked with antithyroid drugs. These assumptions were tested in three ways: 1 ) by comparing the rate of disappearance of radioiodine from the thyroid after a peak value had been reached with the rate of disappearance obtained from the corresponding curve of serum concentration ; 2) by observing the effect on the thyroidal concentration of $\mathrm{I}^{131}$ by administering a large dose of sodium thiocyanate intravenously; and 3) by comparing directly, by means of succes- 


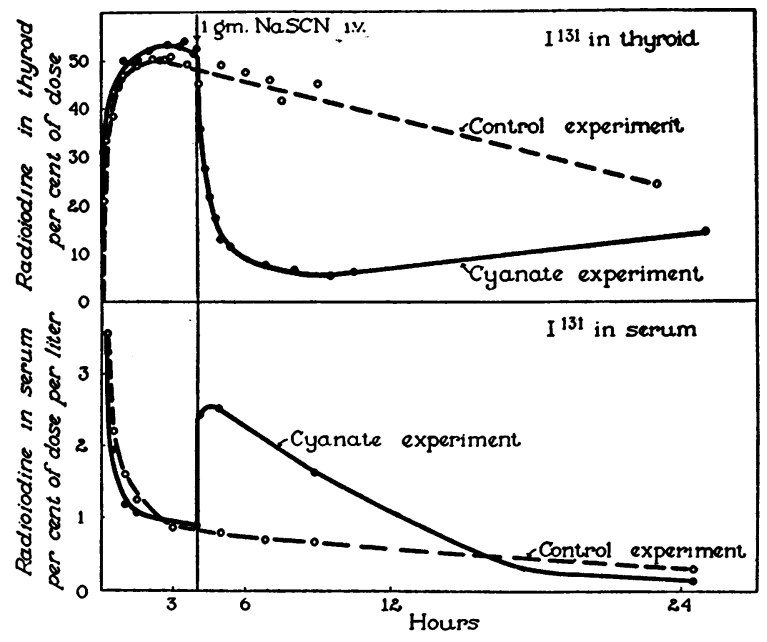

Fig. 6. Effect of Thiocyanate in Discharging IODIDE FROM THE THYROID AFTER A LARGE-CARRIER (5.0 Mg.) Dose of Labeled Iodide in a Patient with ExOPHTHALMIC GOITER (CASE 14)

The control study without thiocyanate was carried out five days before the cyanate study. Note: the notation in the figure should read "thiocyanate experiments" not "cyanate experiments."

sive tracer doses, in a patient with exophthalmic goiter, the effects of small and large doses of carrier iodide with the effects produced by blocking the thyroid with propylthiouracil.

\section{Comparison of large-carrier thyroid curves} with curves of serum concentration

Observations on the serum and the urine were obtained in all the studies here reported, and serum, urine, and thyroid curves for a representative study with $10.0 \mathrm{mg}$. of carrier are shown in Figure 5. The similarity between the thyroidal and serum curves is readily apparent, the principal difference between them being that the thyroidal curve reaches consistently a peak value some time later than the serum curve. In connection with Figure 5 it may be parenthetically observed that the urine curve after administration of $10.0 \mathrm{mg}$. of carrier provides no clue to the temporary retention of a significant fraction of the dose in the thyroid. This is in contrast to the situation when small quantities of carrier are employed and thyroidal accumulation of iodine occurs. In this situation the urine may be used with considerable accuracy to infer the quantitative behavior of the thyroid with respect to iodine accumulation.
The rate of disappearance of radioiodide from the blood may be calculated from the curve of serum concentration in the manner outlined by McConahey and associates (6). Since they follow the same pattern, the thyroidal curves obtained after administration of a large carrier may be used to calculate a disappearance rate in the same manner. Comparison of such rates will provide some measure of whether or not the two curves are in equilibrium with one another, as observed by Taurog, Chaikoff, and Feller (11) in the rat. Disappearance rates calculated in this manner for serum and thyroid are compared in Table IV with disappearance rates calculated from the urinary excretion curve. The rates observed for the thyroid are approximately the same as those for the blood and urine, and therefore it may be inferred that the iodine in the thyroid is in some equilibrium with that in the blood.

\section{The effect of sodium thiocyanate upon the thy- roidal concentration of iodine after a large dose of iodide}

As a control study a patient with recurrent exophthalmic goiter (Case 14) was first given intravenously 300 microcuries of $\mathrm{I}^{181}$ with $5.0 \mathrm{mg}$. of $\mathrm{I}^{127}$ as carrier. Observations were made of thy-

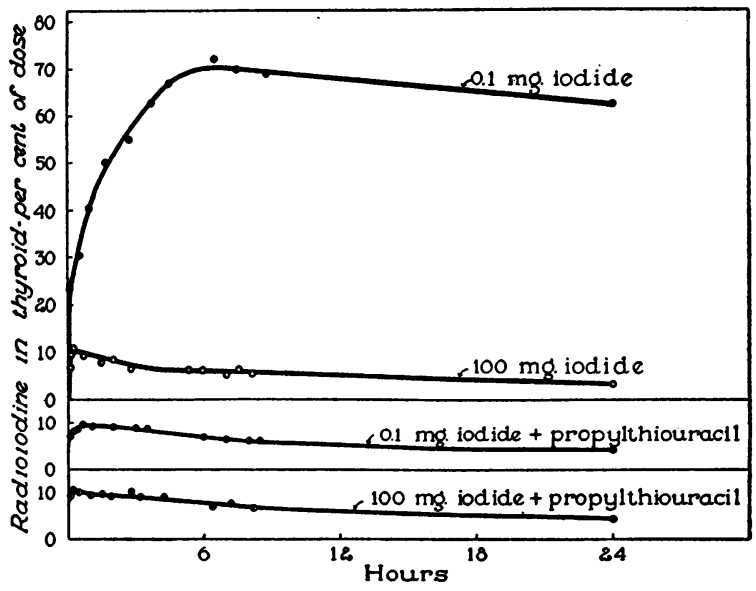

Fig. 7. Comparison of Two Levels of Iodide AdMinistration ( 0.1 AND 100 Mg. OF CARRIER IODIDE I ${ }^{127}$ ) in the Same Patient with Exophthalmic Goiter, First Without and Later With Effective Treatment With Propylthiouracil

It was impossible to show clearly all four curves on the same ordinates because three of the curves were so nearly identical. 
roidal, serum, and urinary concentrations in the usual way. The response was of the large-carrier type. Five days later an identical dose was repeated, and four hours later $1 \mathrm{gm}$. of sodium thiocyanate was injected intravenously. The results are shown in Figure 6 . Thyroidal radioiodine decreased 25 per cent within 10 minutes and 50 per cent within 20 minutes. Of interest is the fact that the curve leveled off at approximately 20 per cent of the preinjection level. A significantly larger amount of radioiodine was in the thyroid after 24 hours despite the probability that a substantial amount of thiocyanate ion remained in circulation. The effect of the thiocyanate ion upon the concentration of radioiodide in the serum was as striking as the change in the thyroid itself, the concentration increasing more than 250 per cent within ten minutes.

\section{Comparison of the thyroidal curves obtained after administration of large carriers and after blocking the thyroid with propylthiouracil}

An additional patient with exophthalmic goiter (Case 15) was studied in the usual way with radioiodine, carrier doses of 0.1 and $100.0 \mathrm{mg}$. of $\mathrm{I}^{127}$ respectively being employed. Following this, the same studies were repeated during treatment with propylthiouracil. Six-tenths of a gm. of propylthiouracil was given 12 hours before each dose of $\mathrm{I}^{131}$. Further doses of $0.2 \mathrm{gm}$. were given one hour before, and three and eight hours after the test. The results of these four studies on this patient are shown in Figure 7. A typical smallcarrier thyroidal accumulation curve was obtained with $0.1 \mathrm{mg}$. of iodide carrier and a typical largecarrier response with $100 \mathrm{mg}$. of iodide. The curve obtained after administraton of $0.1 \mathrm{mg}$. of carrier during treatment with propylthiouracil was so similar to that obtained with $100 \mathrm{mg}$. of $\mathrm{I}^{127}$ alone that it was necessary to plot it on a separate ordinate in order to show the observations distinctly. The fourth curve, that obtained with $100 \mathrm{mg}$. of carrier while the thyroid was blocked with propylthiouracil, also appears to be indistinguishable from the preceding two.

These data suggest that: 1) thyroidal observations after administration of large-carrier doses of iodide measure the same thyroidal function as observations after the thyroid has been blocked with antithyroid drugs; 2) blockage of the rela- tive biosynthetic function as evaluated from acute in vivo studies of $\mathrm{I}^{131}$ accumulation appears equally effective with large doses of iodides or with antithyroid drugs; 3 ) by analogy with the observations on animals, substantially all of the iodine in the blocked thyroid, or in the unblocked thyroid when large quantities of carrier iodide are used, is inorganic iodide and not organically combined iodine ; 4) within the range 0.1 to $100 \mathrm{mg}$. of iodide the administration of iodide does not appear, at least in this case, to modify the magnitude of the iodide-trapping function of the thyroid.

It appears from the foregoing that the specific aspect of thyroidal function which is being observed when tracer doses of $\mathrm{I}^{131}$ with small quantities of carrier $\mathrm{I}^{127}$ are given is distinctly different from that which is observed after administration of large quantities of carrier iodide. In the first instance, for reasons enumerated elsewhere, it is considered that the thyroidal accumulation curve measures primarily the process by which iodine is organically combined to form thyroid hormone. It has been shown elsewhere (5) that this specific aspect of thyroidal function is best evaluated in the human by determining directly thyroidal iodide clearance from observations of thyroidal and serum radioiodine. It may also be evaluated from thyroidal observations alone by calculating the thyroidal accumulation rate. A reasonably accurate index of accumulation rate may be obtained from urinary observations alone, at least in euthyroid and hyperthyroid persons, by calculating the extrarenal disposal rate.

In the case of large-carrier curves, the curve of radioiodine in the thyroid reflects the thyroidal concentration of inorganic iodide, which has been called the thyroidal iodide trap. It is evident from the nature of this function that it cannot be evaluated appropriately in the same manner as the biosynthetic function. Probably the most explicit method for evaluating the iodide trap, first employed by Vanderlaan and Vanderlaan, is expressed in the ratio:

\section{Concentration of iodide/gm. of thyroid tissue Concentration of iodide/cc. of blood serum}

Because it involves thyroid weight, which in clinical studies can be estimated only roughly by palpation, this ratio provides comparatively inexact measurement in patients. A satisfactory expres- 
TABLE V

Evaluation of thyroidal iodide trap

\begin{tabular}{|c|c|c|c|c|c|}
\hline Case & $\begin{array}{c}\text { Basal } \\
\text { metabolic } \\
\text { rate }\end{array}$ & $\begin{array}{l}\text { Estimated } \\
\text { weight of } \\
\text { thyroid }\end{array}$ & $\begin{array}{l}\text { Carrier } \\
\text { iodide }\end{array}$ & $\begin{array}{l}\text { Thyroidal concentrating power* } \\
\left(\frac{\text { conc./gm. thyroid }}{\text { conc./cc. serum }}\right)\end{array}$ & $\left.\begin{array}{c}\text { Thyroidal iodide space } \\
\text { (per cent of dose in thyroid } \\
\text { conc./liters serum }\end{array}\right)$ \\
\hline $\begin{array}{r}3 \\
4 \\
5 \\
8 \\
\\
9 \\
10 \\
13\end{array}$ & $\begin{array}{c}\text { per cent } \\
25 \\
23 \\
24 \\
\\
22 \\
41 \\
40 \\
30\end{array}$ & $\begin{array}{l}g m . \\
40 \\
20 \\
20 \\
\\
40 \\
\\
20 \\
20 \\
20\end{array}$ & $\begin{array}{c}\text { mg. } \\
10 \\
10 \\
10 \dagger \\
(100) \\
10 \\
(500) \\
10 \\
10 \\
10\end{array}$ & $\begin{array}{c}\text { times } \\
134 \\
400 \\
165 \\
(108) \\
250 \\
(54) \\
310 \\
680 \\
540\end{array}$ & $\begin{array}{c}\text { liters } \\
5.3 \\
17.0 \\
3.3 \\
(2.2) \\
10.0 \\
(1.9) \\
6.3 \\
13.4 \\
11.0\end{array}$ \\
\hline Means $\ddagger$ & & & & $354 \pm 49$ & $9.5 \pm 1.8$ \\
\hline
\end{tabular}

* The values for thyroidal and serum I $^{131}$ employed were those corresponding to the time of maximal thyroidal concentration.

$\dagger$ The $10 \mathrm{mg}$. study in Case 5 behaved like an intermediate curve and the calculation used may not be entirely valid in this instance.

$\ddagger$ Not including the values in parentheses.

sion for the magnitude of the iodide trap which avoids the errors inherent in estimating thyroid weight can be obtained with the ratio:

Quantity of iodide in thyroid as per cent of dose Concentration of iodide in serum as per cent/liter determined or interpolated from the actual observations for some specific time. ${ }^{4}$

Expressed in terms of the first ratio, the iodide trap is a concentrating power and the value represents the number of times the concentration of $\mathrm{I}^{131}$ as iodide in the thyroid exceeds that in the serum. In the second ratio, the thyroidal iodide trap is expressed in liters of what might be called thyroidal iodide space. In patients with exophthalmic goiter this space appears to be a substantial part of the total volume of distribution of iodide in the body, and may account for the fact reported elsewhere (5) that rough estimates of the volume of distribution of iodide in patients having exophthalmic goiter, as calculated from curves of serum concentrations of iodide, were higher by 50 per cent than estimates for euthyroid or hypothyroid patients.

Table $\mathrm{V}$ shows the values for these ratios in seven patients receiving large-carrier doses. It is

4 To the extent that the two curves (thyroid and serum) diminish at the same rate, the time selected should not matter so long as it is after the peak value of the thyroid curve has been reached. We have chosen to use the time corresponding to the peak value in the thyroid. of interest that the range of values obtained in patients with exophthalmic goiter is as high as, or higher than, those observed in goitrous rats by Vanderlaan and Vanderlaan (10) or by Taurog, Chaikoff, and Feller (11).

The figures for the $100 \mathrm{mg}$. study in Case 5 and $500 \mathrm{mg}$. study in Case 8 suggest that very large concentrations of iodide may exceed the capacity of the iodide-trapping function and thus appear to suppress it. This may be in keeping with the observation of Stanley and Astwood (9) that administration of $1 \mathrm{gm}$. of potassium iodide produced a discharge of radioiodine from the thyroid comparable to the discharge induced with thiocyanate. The serum iodide concentrations observed during these two studies were of the order of $0.6 \mathrm{mg}$. per $100 \mathrm{cc}$. and $2.6 \mathrm{mg}$. per $100 \mathrm{cc}$. respectively.

It would be helpful to compare the values for iodide trap in cases of exophthalmic goiter with values obtained from normal persons. Unfortunately, we have so far found it technically impossible to obtain accurate information, owing to the fact that in the normal the concentrating power appears to have about the magnitude of that of the normal rat, that is, about 25 to 50 times, or 1 liter or less. At this concentration the quantity of radioactivity observed at $10 \mathrm{~cm}$. over the thyroid with a Geiger counter at the peak of the blood curve or subsequently is only three to five times the cor- 
responding count at the same distance from the thigh. As we have observed empirically that athyrotic persons presumed to have no thyroid function whatever always have a neck-thigh ratio of radioactivity between 2.0 and 3.0 , these values cannot be estimated with any accuracy by the technic we have employed in patients with exophthalmic goiter. This very circumstance, however, suggests, as Stanley and Astwood (9) have previously proposed, that the estimation of the iodide trap, by means of large doses of iodide labeled with $\mathrm{I}^{131}$ and in vivo observations, may prove to be of some value in the study and diagnosis of hyperthyroid states.

The original observations of Hamilton and Soley (7) indicated that normal persons accumulated radioiodine in their thyroids in the same manner after administration of $14 \mathrm{mg}$. of $\mathrm{I}^{127}$ carrier as in subsequent studies employing $0.1 \mathrm{mg}$. of $\mathrm{I}^{127}$ carrier. This is consistent with the findings of Stanley $(12,13)$ that the concentration of iodide in plasma required to suppress the euthyroid gland is much larger than that required to suppress the hyperthyroid gland. Accordingly it is probably incorrect to conclude from our observations of subjects with exophthalmic goiter that the normal human thyroid would behave qualitatively or quantitatively in the same manner.

Lastly the question arises as to whether the data here presented are to be interpreted as showing that large doses of inorganic iodide merely obscure or actually suppress the iodine-accumulating function of the thyroid in exophthalmic goiter. Stanley $(12,13)$ has interpreted in vivo curves similar to ours, and obtained in a similar manner, as indicating that high levels of plasma iodide inhibit iodine accumulation and therefore hormonal biosynthesis in exophthalmic goiter just as they do in the normal rat. However, radioiodine studies involving large quantities of $\mathrm{I}^{\mathbf{1 2 7}}$ suffer from the serious disadvantage of low specific activity. On this account ${ }^{-}$as well as the difficulty of evaluating the neck-thigh ratio mentioned previously, in vivo technics are extremely inaccurate so far as direct measurement of minute proportional amounts of iodine is concerned. There is no reasonable doubt from the data presented that large doses of iodide cause a remarkable suppression of the relative rate of hormonal biosynthesis in proportion to the quantity of iodide available in the circulation. On present evidence, however, we are reluctant to interpret this observation as indicating with certainty that the absolute rate of hormonal biosynthesis is or is not reduced or abolished.

\section{SUM MARY}

1. The renal clearance or renal excretion rate of iodide in patients with exophthalmic goiter was not significantly altered by variations in the quantity of stable (carrier) iodide in the range of 0 to $2.0 \mathrm{gm}$.

2. The relative iodine-accumulating function of the thyroid in patients with exophthalmic goiter was not significantly altered in most instances by increasing the amount of stable carrier from 0 to $0.1 \mathrm{mg}$. Quantities of carrier more than $0.1 \mathrm{mg}$. significantly reduced the relative iodine-accumulating function as measured by extrarenal disposal rate. Quantities of carrier iodide of the order of $1 \mathrm{mg}$. reduced extrarenal disposal rate to euthyroid proportions in the majority of instances; $10 \mathrm{mg}$. or more of carrier in most cases reduced it to negligible proportions.

3. After doses containing carrier iodide in the range 0.001 to $0.1 \mathrm{mg}$. in vivo observations show that $I^{131}$ progressively accumulates in the thyroid in a fairly smooth exponential manner. After doses of $10.0 \mathrm{mg}$. or more of carrier iodide the curve of thyroidal $I^{131}$ rises rapidly to a peak in two to three hours, and thereafter decreases rapidly in amount, paralleling the fall of radioactivity in serum. Doses containing carrier iodide between 0.1 and $10.0 \mathrm{mg}$. result in curves which appear to be intermediate between these extremes.

4. The thyroidal function portrayed with the use of small quantities of carrier iodide $(0.1 \mathrm{mg}$. or less) is considered to be predominantly the utilization of iodine in the biosynthesis of thyroid hormone. The thyroidal function disclosed with the use of large doses of carrier iodide is considered to be the concentration of inorganic iodide within the thyroid, called the iodide-trapping or iodide-concentrating function.

5. The relative biosynthetic function appears to be suppressed by doses of iodide in excess of 0.1 mg. in subjects with exophthalmic goiter. In these subjects, the iodide-trapping function may not appear to be altered with doses in the range 0.1 to $100 \mathrm{mg}$. but may appear to be suppressed by doses of $500 \mathrm{mg}$. or more. The relative bio- 
synthetic function appears to be suppressed or obscured at a serum concentration of inorganic iodide of the order of 5 micrograms per $100 \mathrm{cc}$. or less; the capacity of the trapping function appears to be exceeded at serum concentrations of inorganic iodide of the order of $2 \mathrm{mg}$. per $100 \mathrm{cc}$.

6. The iodide-trapping function of the thyroid can be evaluated in the human subject following high-carrier doses by comparison of the quantity of radioiodine in the thyroid with the concentration in the serum at the same time. The mean ratio of thyroidal content to serum concentration in seven cases of exophthalmic goiter tested disclosed a mean thyroidal iodide space of 9.5 liters. The method here employed was not found suitable for accurate evaluation of the thyroidal iodide-trapping mechanism in euthyroid patients, which appears to be of the order of 1 liter, or less.

\section{BIBLIOGRAPHY}

1. Curtis, G. M., Puppel, I. D., Cole, V. V., and Matthews, N. L., The normal urinary iodine of man. J. Lab. \& Clin. Med., 1937, 22, 1014.

2. Keating, F. R., Jr., Power, M. H., Berkson, Joseph, and Haines, S. F., The urinary excretion of radioiodine in various thyroid states. J. Clin. Invest., 1947, 26, 1138.

3. McConahey, W. M., Keating, F. R., Jr., and Power, M. H., The behavior of radioiodine in the blood. J. Clin. Invest., 1949, 28, 191.

4. Luellen, T. J., Keating, F. R., Jr., Williams, M. M. D,. Berkson, Joseph, Power, M. H., and McConahey, W. M., Relative measurement in vivo of accumulation of radioiodine by the human thyroid gland: comparison with radioactivity in peripheral tissues. J. Clin. Invest., 1949, 28, 207.

5. Keating, F. R., Jr., Wang, J. C., Luellen, T. J., Williams, M. M. D., Power, M. H., and McConahey, W. M., The measurement of the iodine-accumulating function of the human thyroid gland. J. Clin. Invest., 1949, 28, 217.

6. McConahey, W. M., Keating, F. R., Jr., Power, M. $H$. , and Berkson, Joseph, Determination of renal clearance of radioiodine. Unpublished data.

7. Hamilton, J. G., and Soley, M. H., Studies in iodine metabolism of the thyroid gland in situ by the use of radio-iodine in normal subjects and in patients with various types of goiter. Am. J. Physiol., 1940, $131,135$.

8. Hamilton, J. G., The use of radioactive tracers in biology and medicine. Radiology, 1942, 39, 541.

9. Stanley, M. M., and Astwood, E. B., The accumulation of radioactive iodide by the thyroid gland in normal and thyrotoxic subjects and the effects of thiocyanate on its discharge. Endocrinology, 1948, 42, 107.

10. Vanderlaan, J. E., and Vanderlaan, W. P., The iodide concentrating mechanism of the rat thyroid and its inhibition by thiocyanate. Endocrinology, 1947, 40, 403.

11. Taurog, Alvin, Chaikoff, I. L., and Feller, D. D., The mechanism of iodine concentration by the thyroid gland: its nonorganic iodine-binding capacity in the normal and propylthiouracil-treated rat. J. Biol. Chem., 1947, 171, 189.

12. Stanley, M. M., The effect of the iodide ion on thyroid hormone formation in man. (Abstr.) J. Clin. Invest., 1949, 28, 812.

13. Stanley, M. M., The direct estimation of the rate of thyroid hormone formation in man. The effect of the iodide ion on thyroid iodine utilization. $\mathrm{J}$. Clin. Endocrinol., 1949, 9, 941. 